\title{
Direct simulation Monte Carlo method for gas flows in micro-channels with bends with added curvature
}

\author{
Tomáš Tisovskýa,1, Tomáš Vit ${ }^{\mathrm{b}, 1}$ \\ ${ }^{1}$ Department of Power Engineering Equipment, Faculty of Mechanical Engineering, Technical University of Liberec, Czech Republic
}

\begin{abstract}
Gas flows in micro-channels are simulated using an open source Direct Simulation Monte Carlo (DSMC) code dsmcFOAM for general application to rarefied gas flow written within the framework of the open source C++ toolbox called OpenFOAM. Aim of this paper is to investigate the flow in micro-channel with bend with added curvature. Results are compared with flows in channel without added curvature and equivalent straight channel. Effects of micro-channel bend was already thoroughly investigated by White et al. Geometry proposed by White is also used here for refference.
\end{abstract}

\section{Introduction}

Considerable progress in field of miniaturization over recent years, most notably the advancements in development of MEMS (micro-electro-mechanical systems) have led to the need of investigation of gaseous flow in micro-scale geometries.

Gas flow can be modeled either at macroscopic level (Navier-Stokes equations) or at microscopic level.

The Direct Simulation Monte Carlo method, as described by Bird [1], is the dominant numerical method for solving engineering problem involving rarefied gas flow.

Degree of rarefaction of a gas is classified throughout the dimensionless Knudsen number

$$
K n=\frac{\lambda}{L},
$$

where $\lambda$ is mean free path of the gas molecules and $L$ is the characteristic dimension of the flow.

As can be seen from equation (1), high degree of rarefaction can be achieved by either lowering the density of the gas or decreasing characteristic dimension of the fluid region.

The continuum fluid assumption is valid roughly for $K n<0.001$ [1]. In present study, flows with Knudsen number around 0.05 are considered.

For sufficiently high Knudsen numbers, the continuum hypothesis breaks down and solution of the Navies-Stokes equations yields physically incorrect results. Micro-scale flows exhibit many features that are not to be observed in similar cases of macro-scale flows. Most notable difference is that while the continuum solution gives zero velocity at walls (the so called no slip condition), rarefied gas does not behave in this manner and there is a considerable velocity slip at walls. Similarly, there is also temperature jump at walls. Other considerable difference are the non-linear pressure gradients that have been observed in micro-channel flows [3].

Based on $K n$, gas flow can be classified into four regimes [4]

Table 1. Flow classification.

\begin{tabular}{|c|c|}
\hline Regime & Kn \\
\hline Continuum flow & $K n<0.001$ \\
\hline Slip flow & $0.001<K n<0.1$ \\
\hline Transition flow & $0.1<K n<10$ \\
\hline Free molecule flow & $K n>10$ \\
\hline
\end{tabular}

The slip flow can be well modeled with the NavierStokes equations with specific velocity slip and temperature jump boundary conditions. For transition flow, no such approach can be used and methods based on molecular gas dynamics are the only alternative.

Since DSMC is the dominant method for solving rarefied gas flows, there has been many DSMC studies dealing with straight microchannel gas flows $[2,3,7,8$, 9]. However, there is significant lack of data concerning microchannels of more complex geometry. Nevertheless, White et al. [2] used DSMC and carried out series of numerical simulations for flow in microchannels with

\footnotetext{
a Corresponding author: tomas.tisovsky@tul.cz

b Corresponding author: tomas.vit@tul.cz
} 
bends. No work on flow in microchannels with bend with added curvature has been found in available literature.

In this paper, DSMC investigation of flow in microchannel with rounded-off bends is carried out. This research is largely based on work of White et al. Apart from their results regarding micro-channel with bends, they also conducted many simulations in order to validate the dsmcFOAM solver [5] against previously published results.

\section{Direct simulation Monte Carlo}

The only chemical elements which form stable monoatomic molecules at standard conditions are the noble gases. Therefore, to simplify the simulation, working gas used in this paper is argon. As a result, no internal modes (rotational or vibrational) are considered.

\subsection{Physics of micro-scale flow}

Macroscopic properties of a flows at low Knudsen numbers are defined as averages of appropriate molecular quantities.

Important quantity in DSMC is the number density $\mathrm{n}$ which gives the number of molecules per unit volume. This can be calculated from the ideal gas law if pressure and temperature are known. Mass of individual molecules is denoted $m$.

Results will be viewed in terms of pressure, mean (macroscopic) velocity and wall shear stress. These quantities are calculated at each time step by following relations and then averaged over large number of samples. In present study, about one million samples were used.

\subsubsection{Calculating flow properties}

Each molecule in gas has velocity $\boldsymbol{c}$. By averaging over large number of molecules, mean molecular velocity $\overline{\boldsymbol{c}}$ can be obtained. Mean molecular velocity is also the macroscopic stream velocity $\boldsymbol{c}_{\mathbf{0}}$. From this, the random thermal velocity component of each molecule can be obtained as

$$
c^{\prime}=c-c_{0}
$$

Scalar pressure tensor can be defined as

$$
p=\frac{1}{3} \rho \overline{\boldsymbol{c}^{\prime 2}} \text {. }
$$

Finally, the viscous stress tensor is defined as

$$
\tau_{i j}=\rho \overline{c^{\prime}{ }_{l} c^{\prime}}-\delta_{i j} p
$$

\subsection{Modeling physical effects in DSMC}

To model rarefied gas flow, it is necessary to compute typical intermolecular collisions and molecule-surface interactions.

In order to perform Direct Simulation Monte Carlo, computation geometry of the flow domain needs to be defined which is then divided into cells. Since the mesh cells are used mainly to determine potential collision partner for given cell and otherwise have no effect to the computation, requirements for cell quality (measured most notably by skewness, non-orthogonality and aspect ratio) are, to some extent, not of concern. Typical cell size of the mesh should be a fraction of the mean free path. Similarly, the time step should be a fraction of the mean collision time of the gas molecules. [2] It is the generally low value of the time step that represents the greatest drawback for DSMC with respect to the computational effort needed to arrive to the converged solution.

Basic quantities associated with molecular model are the number of molecules per unit volume and mass, size (diameter) of the molecules, velocity and internal state of each molecule in the system. Those parameters are related to mean free path and collision frequency so that distance and time scales of the effects due to collisions can be evaluated.

\subsection{Simulation procedure}

The DSMC method is a particle method in which each simulated particle represents large number of real gas molecules. The physics of the gas is captured by uncoupling the motion of the particles from their collisions. Collisions between particles are evaluated stochastically after all particles have been moved. Collision then cause discrete changes in individual particles velocities and energies. The algorithm of DSMC is presented in figure 1.

Particles are moved through computational domain during each time step according to their individual velocities and the time step. In the absence of external force field, particles move in straight lines. If $2 \mathrm{D}$ flow is to be simulated, individual particle displacement in homogeneous direction is simply ignored.

Particles which cross boundary of the flow are either removed from the simulation or reflected back into the domain.

Upcoming collision step requires that the particles are grouped into the cells of the computational grid. Particles need to be newly indexed after each move.

Collisions are calculated statistically between particles residing in the same computational cell. Particles are paired in each cell to form a list of possible collision pairs. An accept-reject method is used to determine which pairs will actually collide. Probability of a collision occurring is dependent on the collision model and selection scheme being employed, and is the function of the relative velocity, collision cross section and gas density in that cell. Probability is also proportional to the time step and particle weight. The collision between two particles is accepted if the probability is greater than a random number.

Number of pairs tested and the collision probabilities are such that the average collision rate is equal to that given by the kinetic theory. In OpenFOAM, the No Time Counter method is used. 
Once a pair of particles has been selected for collision, momentum and energy are exchanged (they are, of course, conserved in each collision). Actual trajectories and dynamics of the particles are not considered when determining mechanics of the collision. Post collision properties are generated statistically. Variable hard sphere (VHS) model is used to model individual molecules.

Momentum and translational energy transfer is considered in the center of mass frame of reference. Velocity of the center of mass is held constant to conserve momentum while the magnitude of relative velocity may be changed as a result of internal energy transfer. For VHS collision model, scattering angle is isotropic.

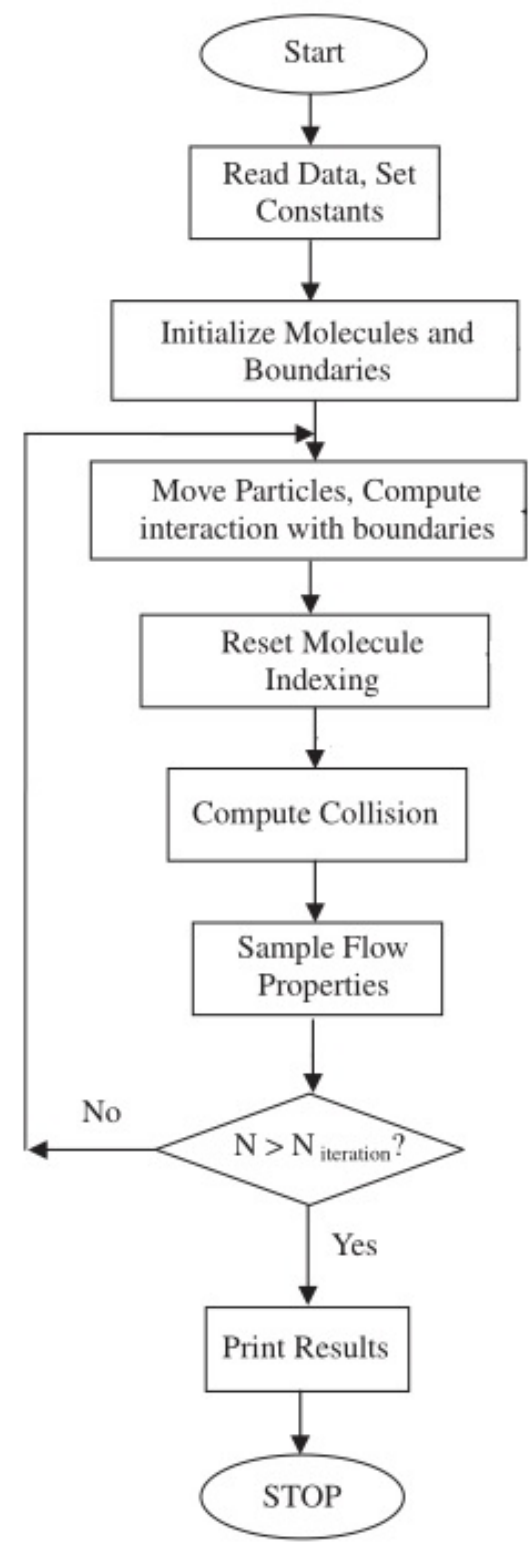

Fig. 1. DSMC algorithm

Macroscopic properties of a gas are local averages of the properties of molecules of the gas. Since DSMC directly simulates the distribution function, flow field results can be determined by sampling particle properties and calculating the moments of sampled data. Computational grid is used to group particles for the purpose of sampling their properties. Flow field properties are calculated at each cell.

Calculation of smooth flow properties requires a large sample size to accurately model the distribution function. Magnitude of statistical fluctuations varies according to the inverse square root of sample size. Real gas is also subject to these fluctuations, but the magnitudes are much smaller due to the sample size which is much larger than the one available in simulations. It is generally necessary to sample over a number of iterations to increase the sample size and therefore reduce fluctuations.

Flows in micro-channels occur generally roughly at normal conditions (i.e. at pressures around atmospheric and at temperatures around $300 \mathrm{~K}$ ). Individual particles have therefore thermal velocities orders of magnitude larger than the overall average velocity (i.e. stream velocity). This makes it difficult to compute macroscopic properties of the flow accurately. Generally, many samples are needed and the DSMC method is expensive.

\subsubsection{Pressure boundary conditions}

The problem with DSMC is, as for virtually any other method for simulating real flow of some fluid, in boundary conditions. The velocity profiles at the inlet and outlet are not known due to current experimental limitations at the micro-scale. Usually, pressure and temperature at inlet and pressure at outlet are the only known thermo-mechanical properties at the boundaries.

Employed pressure inlet boundary condition was the one suggested by Wang [7]. In this approach, income macroscopic velocity for new particles is computed from statistical macroscopic velocity obtained from particles in cells near the inlet boundary. For the inlet face perpendicular to $x$ axis, this gives

$$
\left(c_{0 x_{i n}}\right)_{j}=\left(c_{0 x}\right)_{j}+\frac{p_{\text {in }}-p_{j}}{\rho a} .
$$

Here, $c_{0 x}$ is the $\mathrm{x}$ component of macroscopic stream velocity and $\mathrm{a}$ is the speed of sound.

Velocity component perpendicular to inlet face is

$$
\left(c_{0 y_{i n}}\right)_{j}=\left(c_{0 y}\right)_{j}
$$

To determine inlet number flux density, number density corresponding to inlet pressure is also needed. This is given by ideal gas equation of state

$$
n_{\text {in }}=\frac{p_{\text {in }}}{k T_{\text {in }}} .
$$

Outlet boundary condition is of the form suggested by Liou and Fang $[8,9]$. Density is

$$
\left(\rho_{\text {out }}\right)_{j}=\rho_{j}+\frac{p_{\text {out }}-p_{j}}{a^{2}} .
$$

Since the density is just number density multiplied by mass of one molecule, equation (8) is the equation for 
number density. Considering again the boundary face perpendicular to direction of flow, velocity components are

$$
\begin{gathered}
\left(c_{0 x_{\text {out }}}\right)_{j}=\left(c_{0 x}\right)_{j}+\frac{p_{j}-p_{\text {out }}}{\rho a}, \\
\left(c_{0 y_{\text {out }}}\right)_{j}=\left(c_{0 y}\right)_{j} .
\end{gathered}
$$

And finally, temperature is given by equation of state of ideal gas

$$
\left(T_{\text {out }}\right)_{j}=\frac{p_{\text {out }}}{r\left(\rho_{\text {out }}\right)_{j}} .
$$

Modification of above equations to boundary faces perpendicular to y axis is straight forward.

\section{Micro-channels with rounded bends}

Adding curvature to the bends enables investigation of the effects of competing concave and convex curvature on the flow.

Curved surface of a channel is regarded convex if all the line segments between each pair of points belonging to the surface intersect the interior of the channel. If this condition is not satisfied, the curved surface is concave. Concave surfaces cause the reflected molecules to spread (i.e. diverge) while convex surfaces cause them to meet (i.e. converge).

Diverging character of a reflection in collision between molecule and concave channel surface, as well as in intermolecular collision between two molecules, is the cause of chaos in hard sphere system. In such reflection, small changes in initial trajectories, caused by finite precision of our computers, are magnified at each collision. This justifies the collisions to be calculated statistically between particles residing in the same computational cell.

\subsection{Setting up the simulation}

As in work of White et al. [2], the straight channel length $\mathrm{L}$ is $15 \mu \mathrm{m}$ and its width $\mathrm{B}$ is $1 \mu \mathrm{m}$. Concequently the bends and round offs are added in a way that the channels volume and surface area do not change.

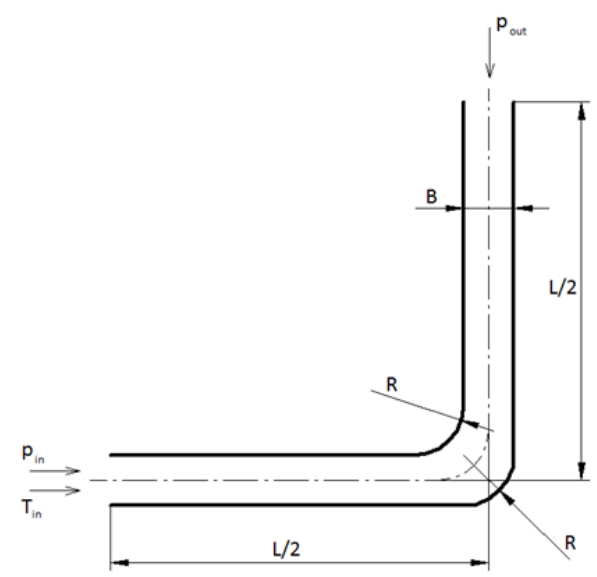

Fig. 2. Micro-channel with rounded-off bend
Geometry of channel with rounded-off bend is shown in figure 2. For future reference and according to figure 2, wall with bend with convex curvature will be reffered to as lower wall, similarly, wall with bend with concave curvature will be reffered to as upper wall.

The working gas is argon and the inlet gas temperature and wall temperature are both $300 \mathrm{~K}$. The walls of the channels are modeled as fully diffuse boundaries.

Computational domain was also meshed in accordance with the work of White. It is recommended that the cell size in DSMC is less than mean free path of the gas molecules. However, it is possible to use elongated cell sizes in the streamwise direction of microchannel flows [2], where macroscopic gradients are relatively small. In channels with bend, the corner region is meshed with cells of sizes smaller than mean free path in both directions.

Value of mean free path is needed to determine flow regime. For VHS molecules, mean free path is [1]

$$
\lambda=\frac{2(5-2 \omega)(7-2 \omega)}{15} \sqrt{\frac{\rho}{2 \pi n k T}} \frac{\mu}{\rho},
$$

where $\omega$ is the viscosity idex of the gas.

From this expression and from the characteristic dimension of the problem, which is here the width of the channel, inlet and outlet Knudsen numbers can be determined.

Two cases were modeled, one partially (at inlet) in slip regime and partially (at outlet) at transition regime. Second one is fully in transition regime.

Table 2. Simulated cases

\begin{tabular}{|c|c|c|c|c|}
\hline Case & $\boldsymbol{p}_{\text {in }}[\mathbf{P a}]$ & $\boldsymbol{p}_{\text {out }}[\mathbf{P a}]$ & $\begin{array}{c}K n_{\text {in }} \\
{[\mathbf{1}]}\end{array}$ & $\begin{array}{c}\boldsymbol{K} \boldsymbol{n}_{\text {out }} \\
{[\mathbf{1 1}]}\end{array}$ \\
\hline Case 1 & 100,000 & 33,333 & 0.055 & 0.158 \\
\hline Case 2 & 18,500 & 6,166 & 0.300 & 0.862 \\
\hline
\end{tabular}

Different computational meshes need to be used for Case 1 and Case 2.

\subsection{Results}

Averaged flow fields were obtained for four geometries, each with the boundary conditions corresponding to case 1 and case 2 . As a result, 8 numerical simulations were performed and results were sampled from approximately one milion samples. It should be noted that computational meshes need to be adjusted in accordance to given set of boundary conditions. Consequently, 8 computational meshes were created.

\subsubsection{Mach number}

Mach number was calculated based on averaged channel centre-line velocity and on speed of sound of perfect gas. It is found that local Mach number decreases in bend 
region and the decrease is more significant as the curvature of the bend gets smaller. Maximum decrease is found for sharp bend.

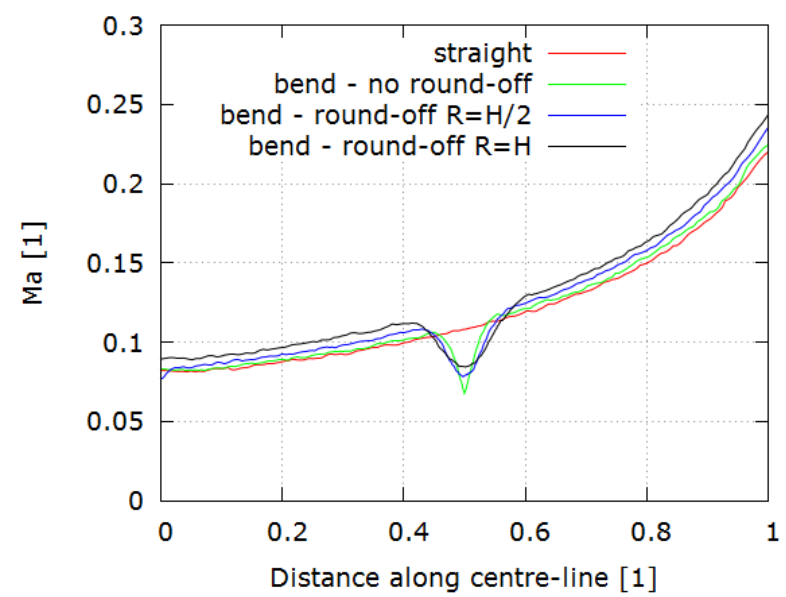

a) Case $1\left(K n_{\text {in }}=0.055\right)$

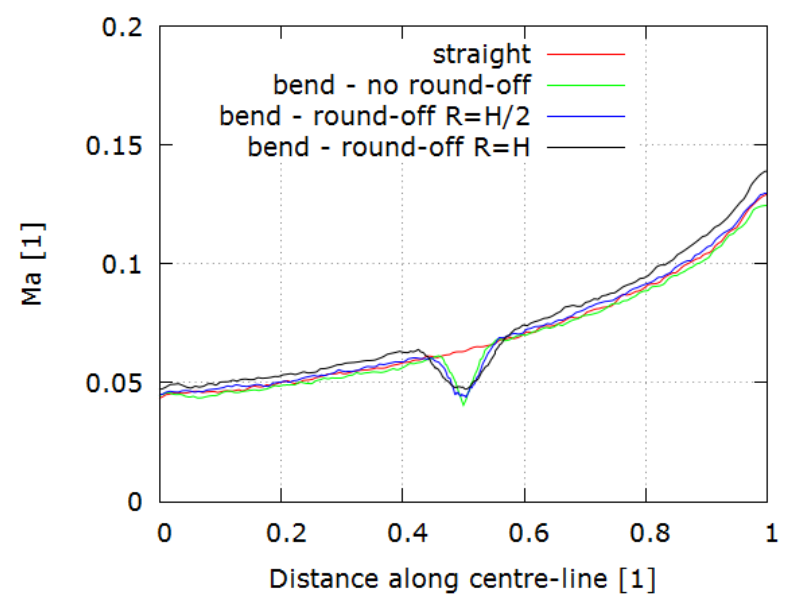

b) Case $2\left(K n_{\text {in }}=0.300\right)$

Fig. 3. Mach number centre-line profiles

\subsubsection{Pressure}

Pressure profiles along channel centre-line closely resemble linear profile. The deviation from linear distribution is plotted in figure 4 and it is greater for larger Knudsen number flows as the rarefaction effects start to dominate over the compressibility effects. At the middle point of the centre-line, the pressure deviations for channels with bends exhibit significant changes. Those are the results of changes in velocity due to the channel geometry (as shown in figure 3 ) and are therefore mostly compressibility effects. The other effect which influences the pressure jump is the flow separation, which can occur in some cases and which lowers the effective cross-section of the flow in corner region. However, this effect is not significant and does not occur for all considered cases (as is demonstrated further).
The pressure jumps for channels with rounded-off bends are greater than in cases of sharp bends and are smoothed of to larger region of space.

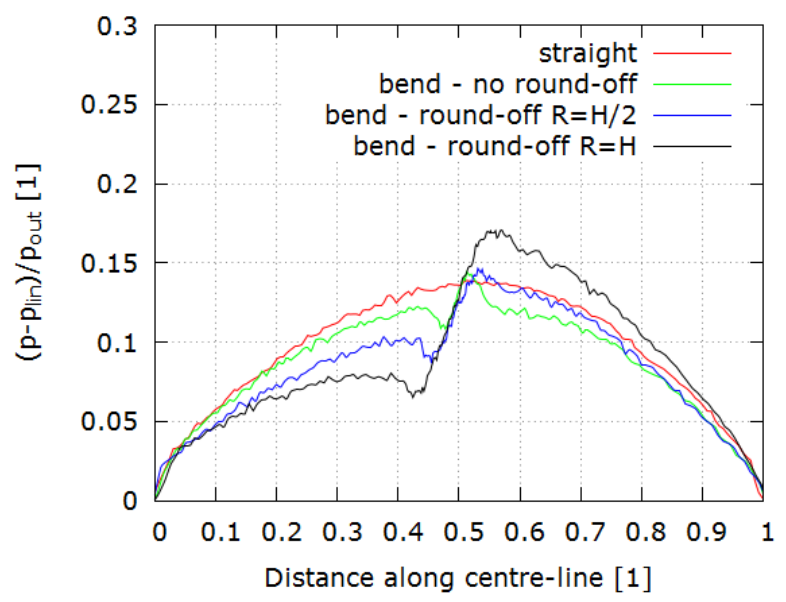

a) Case $1\left(K n_{\text {in }}=0.055\right)$

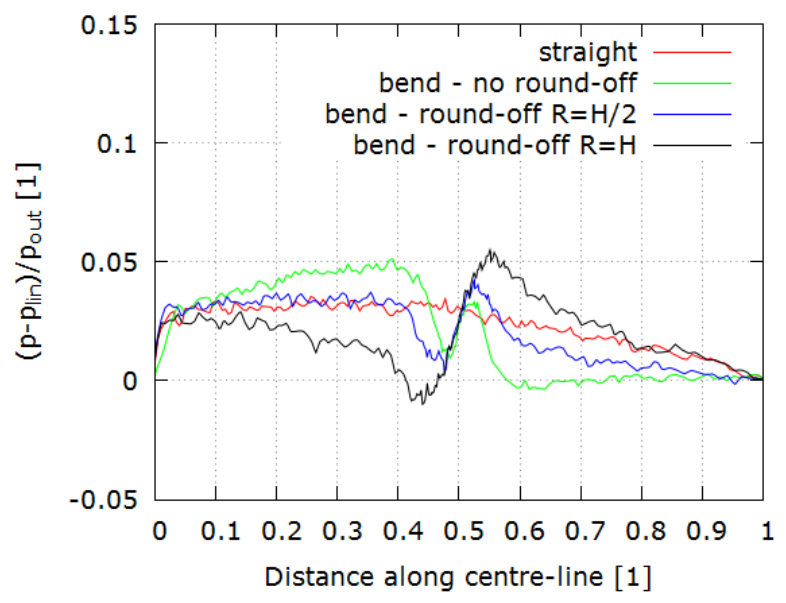

b) Case $2\left(K n_{\text {in }}=0.300\right)$

Fig. 4. Deviation of pressure distribution from linear profile

\subsubsection{Wall shear stress}

Averaged shear stresses in channels with bends are compared with the averaged shear stress in corresponding straight channel. For both cases in consideration, channel with sharp bend exhibited lower shear stress compared to straight channel. With added curvature, the shear stress increased above the value for straight channel.

Care must be taken for the averaging to be taken as weighted average to acount for different width of computational cells in region of bend as compared to rest of the geometry.

Actual shear stresses profiles along both walls for both cases are shown in figures 5 and 6 with $5 \mathrm{a}$ and $6 \mathrm{a}$ corresponding to lower walls and $5 \mathrm{~b}$ and $6 \mathrm{~b}$ corresponding to upper walls. 
Table 3. Normalized average shear stress $\frac{\tau}{\tau_{\text {straight }}}$

\begin{tabular}{|l|l|l|l|}
\hline $\boldsymbol{K} \boldsymbol{n}_{\text {in }}$ & $R=0$ & $R=H / 2$ & $R=H$ \\
\hline 0.055 & 0.979 & 1.011 & 1.041 \\
\hline 0.300 & 0.946 & 0.980 & 1.019 \\
\hline
\end{tabular}

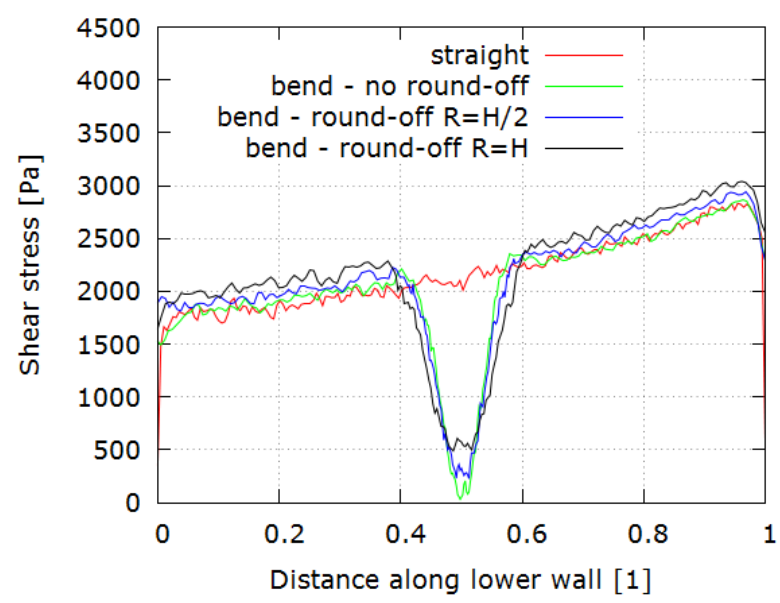

a) wall with convex curvature (lower wall)

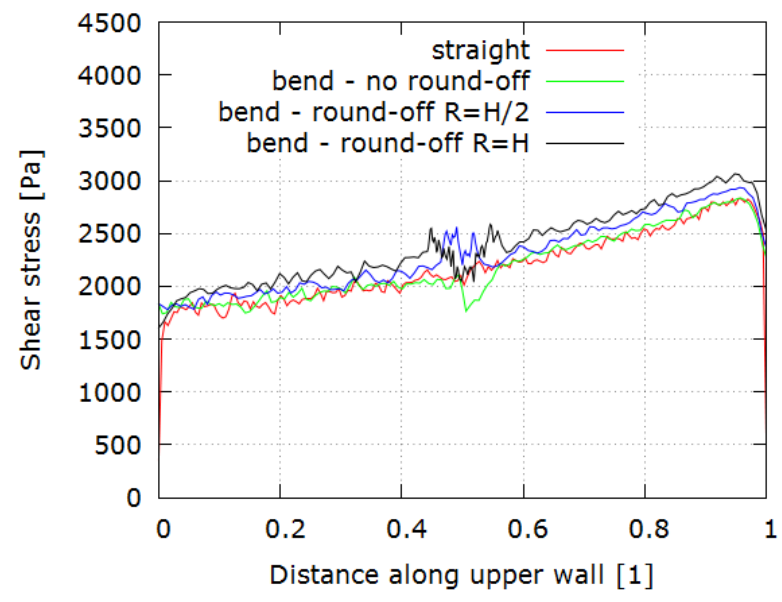

b) wall with concave curvature (upper wall)

Fig. 5. Wall shear stress for Case $1\left(K n_{\text {in }}=0.055\right)$

Case of slip to transition regime exhibits significant drops in wall shear stress at lower wall in region of the bend. Consequently, if there is a reduction in averaged wall shear stress, most of this reduction is achieved there. As curvature is added to the bend, the decrease in wall shear stress can become insufficient to compensate for the modest increase in wall shear stress which is evident for channels with bends along straight parts of its walls and which becomes more significant as the radius of curvature increases. Apart from this increase, there is also little bit more significant increase in regions close to the edges of curvature at upper wall. Interestingly, this increase becomes significant only for large enough curvatures. For sharp bend, there is a decrease in wall shear stress in this region.

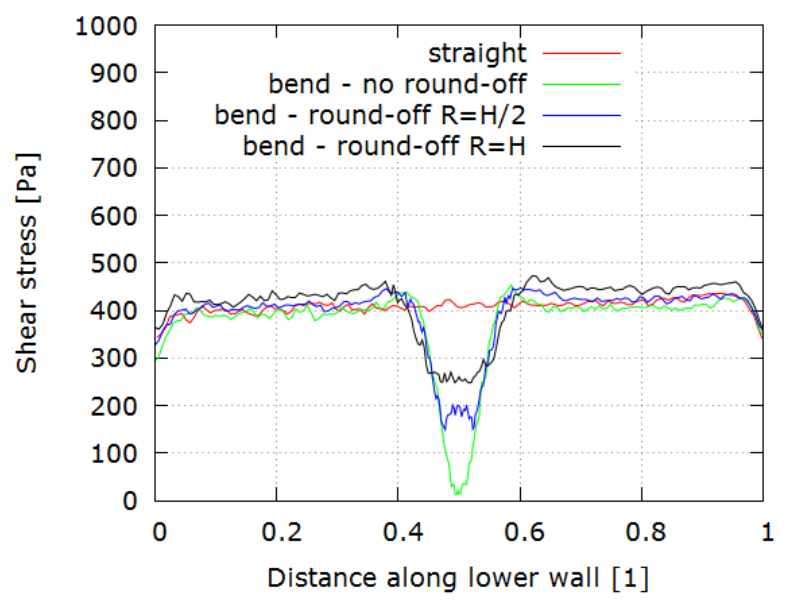

a) wall with convex curvature (lower wall)

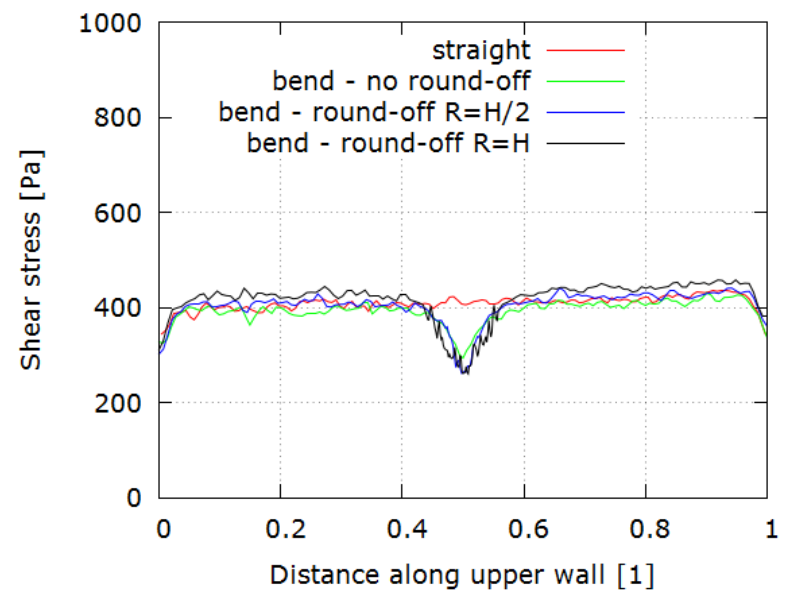

b) wall with concave curvature (upper wall)

Fig. 6. Wall shear stress for Case $2\left(K n_{\text {in }}=0.300\right)$

On the other hand, case of fully transition regime exhibits significant wall shear stress drops in regions of the bend for both upper and lower wall. As in case of slip to transition regime, reduction of wall shear stress can only be achieved if the decrease in bend region compensates for the increase along the straight parts of walls of channel with bend. Since this increase once again becomes more significant with increasing radius of curvature of the bend, there is once again a threshold of radius of curvature above which is the averaged wall shear stress greater than in corresponding case of straight channel. For fully transition regime, this radius of curvature is larger than in case of slip to transition regime.

Since wall shear stress at upper wall for case of slip to transition regime for sharp bend has the same character as wall shear stress at upper wall for case of fully transition regime for bends with added curvature, it can be predicted that, for sufficiently large radius of 
curvature, wall shear stress profile similar to that of slip to transition regime with curved bend may be achieved in fully transition regime for sufficiently large radius of curvature.

\subsubsection{Slip velocity}

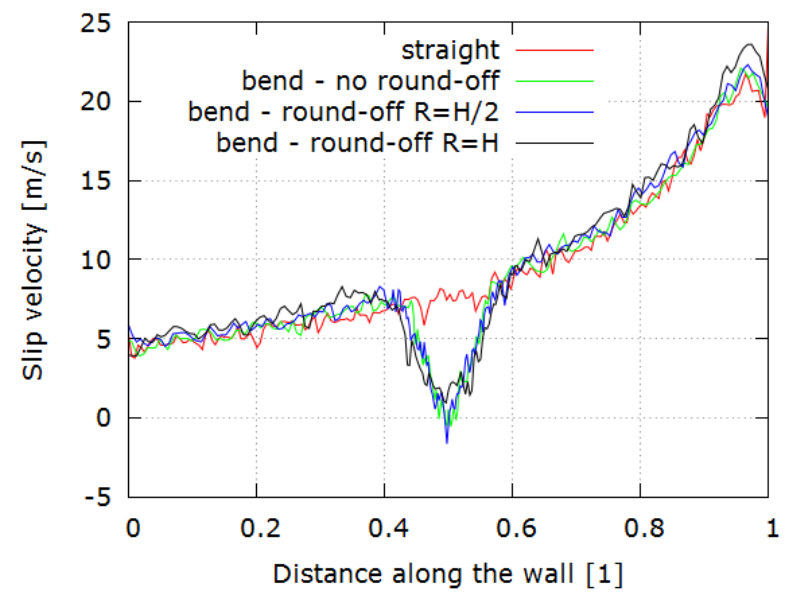

a) wall with convex curvature (lower wall)

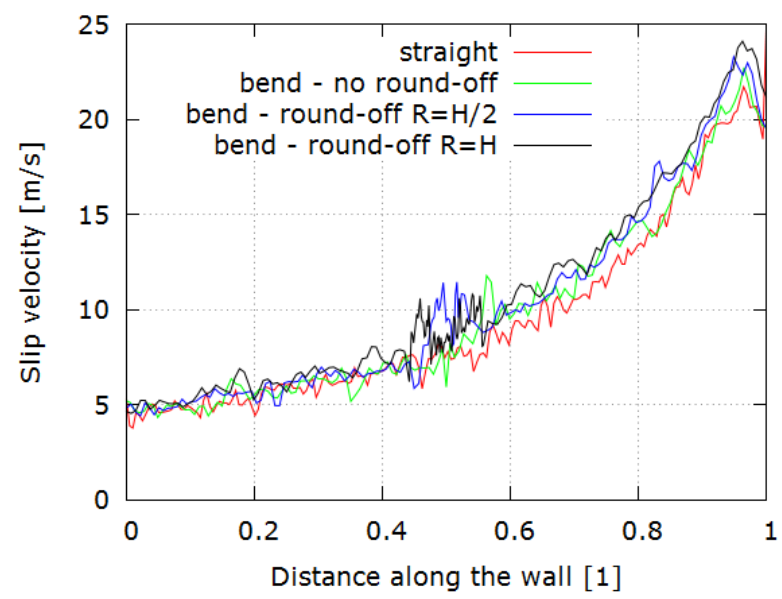

b) wall with concave curvature (upper wall)

Fig. 7. Slip velocity for Case $1\left(K n_{\text {in }}=0.055\right)$

Figures 7 and 8 show slip velocity along both the channel walls for case 1 and case 2. Possitive values of slip velocity are those in direction from inlet to outlet (i.e. in the negative direction of the pressure gradient along the channel). Case of $K n_{\text {in }}=0.055$ for sharp bend and for $R=H / 2$ and case of $K n_{\text {in }}=0.300$ for sharp bend both exhibit slip velocity drop below zero for the wall with convex curvature in region of the bend. This suggests existence of a small region of separated flow in a form of vortex.

Slip velocity along the wall with concave curvature shows modest increase in the region of the bend for the case of more pressurized flow $\left(K n_{i n}=0.055\right)$ and modest decrease in the same region in case of less pressurized flow $\left(K n_{\text {in }}=0.300\right)$.
Furthermore, there is strong correlation between slip velocity and corresponding wall shear stress.

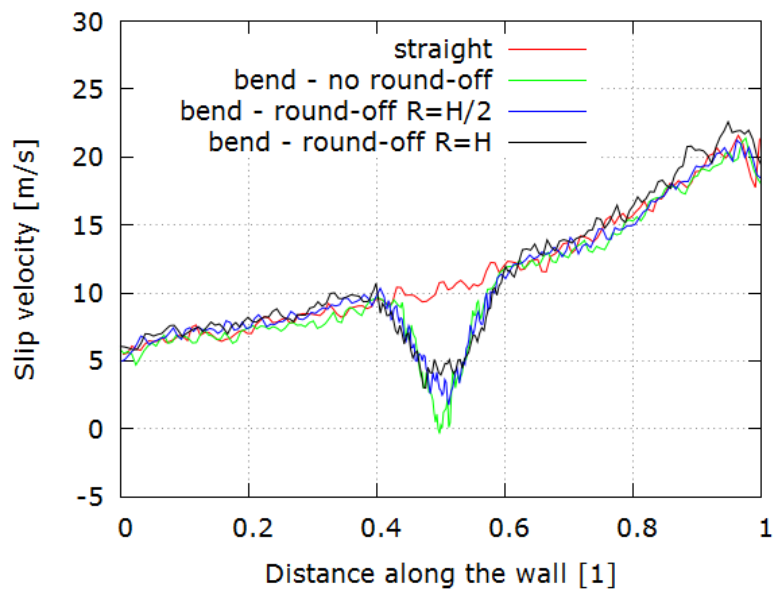

c) wall with convex curvature (lower wall)

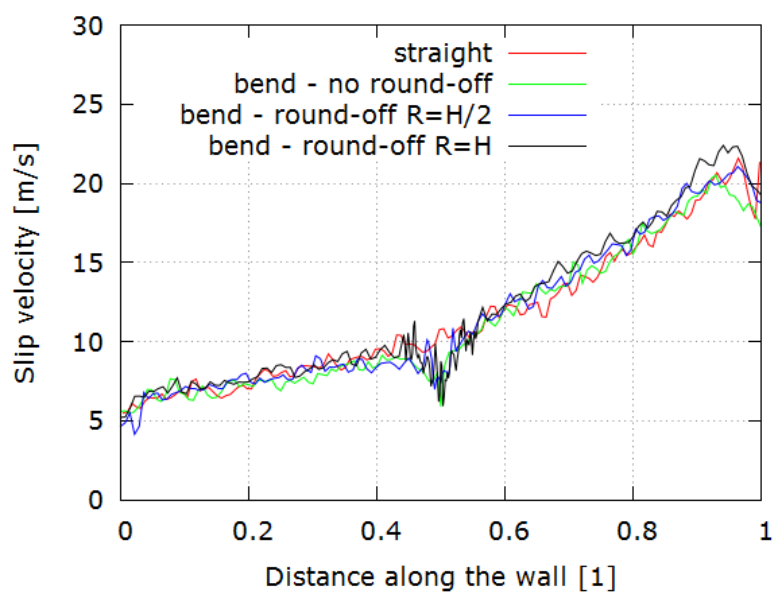

d) wall with concave curvature (upper wall)

Fig. 8. Slip velocity for Case $1\left(K n_{\text {in }}=0.300\right)$

\subsubsection{Mass flow rate}

To investigate the effect of rounded-off bends to the mass-carrying capacity of the microchannel, mass flow rates of particular microchannels are normalized with respect to the mass flow rate through corresponding straight microchannel.

It was shown by White [2] that based on boundary conditions, adding bend to microchannel can lead to either increase or decrease of mass flow rate through the microchannel. In this paper, curvature is added to both the above cases and it is found that for investigated cases, this always results in increase of mass flow rate as compared to straight channel. This holds also for case where sharp bend results in decrease in mass flow rate.

Furthermore, it was shown that microchannels with bend in fully slip regime (which were not modeled here) can also exhibit mass flow rate enhancement. This result was obtained separately with DSMC [2], with lattice Boltzmann method [10] and also with continuum fluid 
dynamic simulation with specialized boundary conditions [11].

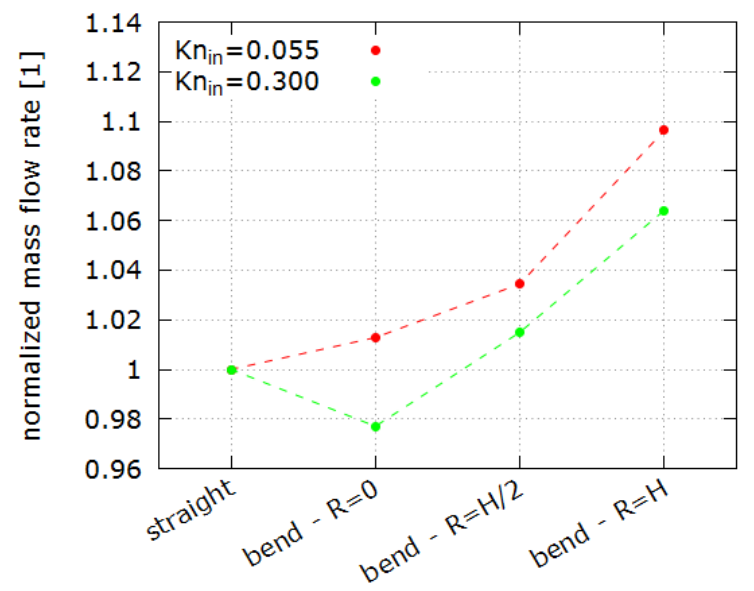

Fig. 9. Mass flow rate through microchannels

Comparing table 3 with figure 9 shows that while the averaged wall shear stress of microchannel with sharp bend is below the corresponding value for straight microchannel, the mass flow rate through the microchannel can be either greater or smaller as compared to that of straight microchannel.

\section{Conclusions}

The investigation of pressure driven flows in microchannels with bends with added curvature was performed for the case of flow in fully transition regime and for the case of flow in slip to transition regime. Each of the cases investigated consisted of simulations of straight channel, channel with sharp bend and two channels with curved bends. In total, 8 DSMC simulations were performed and special care was taken to model the bend region with cells sufficiently small to capture possible regions of separated flow. Such region was subsequently confirmed even for one of the cases with curved bend.

Most notably, it was found that adding curvature to the bend can eventually lead to increase in mass flow rate through the channel even in the case where, for the same boundary conditions, adding sharp bend would result in mass flow rate decrease. This is an important result which can prove valuable in practical applications such as design of MEMS devices.

For future work, more throughout investigation of gas flow in microchannels should be conducted. Especially in fully slip regime which present paper does not deal with. This regime offers the possibility to model with both the molecular dynamics methods and with continuum fluid dynamic methods with specialized boundary conditions. In this case DSMC method is more reliable but also more computationally demanding than simulation of more rarefied regimes. Apart from this, greater curvatures should also be added to the bend to obtain better picture of the character of the change in mass flow rate.

\section{Acknowledgement}

We gratefully acknowledge the support of the Grant Agency of the Czech Republic (Project No. 16-16596).

\section{References}

[1] Bird G. A. Molecular gas dynamics and the direct simulation of gas flows. New York: Oxford Science Publications, Oxford University Press Inc., 1994.

[2] White C., Borg M. K., Scanlon T. J., Reese J. M. A DSMC Investigation of Gas Flows in Microchannels with Bends. Journal of Computers \& Fluids 2012.

[3] Arkilic E. B. Measurement of the Mass Flow and Tangential Momentum Accommodation Coecient in Silicon Micromachined Channels. Ph.D. Thesis. Massachusetts Institute of Technology, Cambridge 1997.

[4] Tsien H. Superaerodynamics, The Mechanics of Rarefied Gas. Journal of the Aeronautical Sciences 13:653-664, 1946.

[5] Micro \& Nano Flows for Engineering, dsmcFOAM [Computer code]. Available at http://www.micronanoflows.ac.uk/

[6] OpenFOAM Foundation, 2016. Available at http://www.openfoam.org/

[7] Wang M, Li Z. Simulations for gas flows in microgeometries using the direct simulation Monte Carlo method. International Journal of Heat and Fluid Flow 2004.

[8] Liou WW, Fang Y. Heat transfer in microchannel devices using DSMC.

Journal of Microelectromechanical Systems 2001.

[9] Fang Y, Liou WW. Computations of the flow and heat transfer in microdevices using DSMC with implicit boundary conditions. Journal of Heat Transfer 2002.

[10] Agrawal A, Djenidi L, Agrawal A. Simulation of gas flow in microchannels with a single ninetydegree bend. Computational Fluids 2009.

[11] Rovenskaya O, Croce G. Numerical analysis of rarefaction and compressibility effects in bent microchannels. ASME Conference Proceeding 2010. 